\title{
Adaptive Multi-Modal Sensors
}

\author{
Kyle I. Harrington*, Hava T. Siegelmann ${ }^{\dagger}$ \\ *School of Cognitive Science \\ Hampshire College, Amherst, USA \\ 'Department of Computer Science \\ University of Massachusetts, Amherst, USA \\ kharrington@hampshire.edu, hava@cs.umass.edu
}

Real world applications of robotics and artificial intelligence sometimes require input from unknown environments (Pederson, 2001). For such cases an internal representation of the environment must be created on-the-fly. The use of multi-modal sensors allows general solutions to be used for various classes of unknown environments. By adaptively changing the modality of sensors it is possible to, in many cases, reduce the number of sensors necessary to adequately sense the environment, as well as reduce the amount of bandwidth used by sensors.

Here we present a self-constructive approach to developing a multi-cellular artificial organism capable of adjusting sensor modalities based upon sensory input from a dynamic environment (Fig. 1). The results demonstrate the robustness of this system within an environment of which the organism has no a priori knowledge.

\section{Organism Design}

The multi-cellular organism exists in a 2.5D lattice (Fig. 1a.), where the half dimension represents the ability of cells to stack. Four cell types are used, root stem cells, structural, sensor stem, and sensor cells. A root stem cell can differentiate into root stem cells, structural and sensor stem cells, and a sensor stem cell can
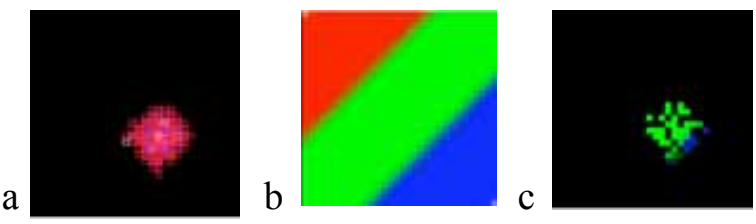

Fig. 1. Three views of the system. (a) the cellular organism (b) the environment to be sensed (c) the sensor activity within the organism for the exact location on (b) differentiate into sensor stem cells and sensor cells. We use chemical diffusion to regulate cell differentiation, division, and migration, in a way similar to Eggenberger's (1997) use of transcription factors, and the use of gases within a GasNet (Husbands et al., 1998).

The organism is initialized as a single root stem cell, then iteratively grows via cell division until it reaches equilibrium. Final size and steps until equilibrium are determined by both diffusion of growth chemicals and the differentiation criteria. The self-constructive growth allows the organism to self-repair. If cells are removed from the organism; diffusion causes the organism's chemical levels to decrease below equilibrium, causing division to resume

\begin{tabular}{|l|l|l|l|l|l|l|l|l|l|l|}
\hline & $\mathbf{1}$ & $\mathbf{2}$ & $\mathbf{3}$ & $\mathbf{4}$ & $\mathbf{5}$ & $\mathbf{6}$ & $\mathbf{7}$ & $\mathbf{8}$ & $\mathbf{9}$ & $\mathbf{1 0}$ \\
\hline S1 & R & G & R & G & R & R & G & R & G & R \\
\hline S2 & R & B & B & B & R & B & R & B & B & B \\
\hline S3 & G & G & R & G & G & G & G & G & G & G \\
\hline S4 & R & R & R & R & R & R & R & G & G & G \\
\hline S5 & R & G & R & G & R & G & G & R & G & R \\
\hline
\end{tabular}

Fig. 2. Sensors tend to focus on two modes, and alternate between the two based upon which is more frequent. 
Each sensor is capable of being in one mode at a time. For each active sensing mode, $\mathrm{A}$, there are two chemicals that act as sensory memory, $C_{S A S}$, short-term and $C_{S A L}$, long-term memory, the former with a higher diffusion coefficient than the latter. These chemicals are produced by,

$$
C_{S A S}=D_{S A L} * S_{A} \quad C_{S A L}=D_{S A S} * S_{A}
$$

where, $S_{A}$ is the activation value of the sensor for mode A. The active sensing mode switches if, either $C_{S A S}<C_{S A L}$,or $C_{S A L}<\Theta_{S A}$, where $\Theta_{S A}$ determines the minimum amount of activity to stay in mode A. For us each sensor was capable of three sensory modalities, red, green, and blue.

\section{Conclusions}

The mean number of sensors in each modality was recorded across a run of 10 organisms. Each run was 60,000 steps. After 40,000 steps $20 \%$ of the cells were removed to demonstrate self-repair. The rapid change between modalities in Figure 2 is the feature of our organism that allows it to properly adapt to its environment.

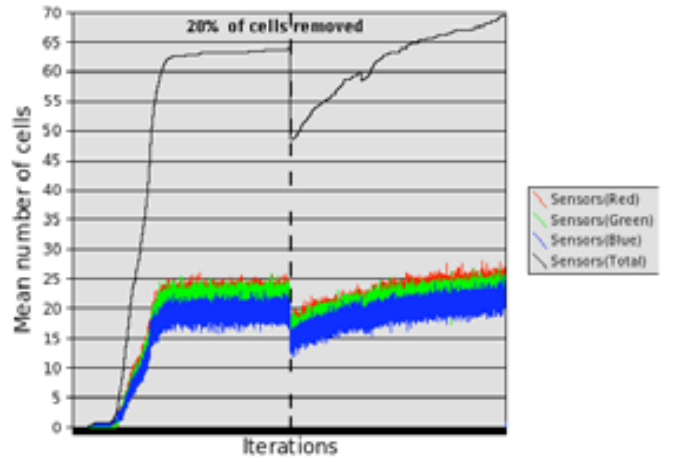

Fig. 3. The top line is the total number of sensors and the bottom are the division of active modalities.
Figure 3 presents results supporting our organism's capability of adapting its sensors to the distribution of color within the environment by maintaining a distribution of sensor modes which is representative of the environment.

Future research will examine the utility of interpolating sensor values from the sensor chemical memory in order to develop a more complete internal representation of the environment.

\section{References}

1. Pedersen, L., Wagner, M., Apostolopoulos, D., Whittaker, W.R.. Autonomous robotic meteorite identification in Antarctica (Proceedings 2001 ICRA. IEEE International Conference on Robotics and Automation, 2001)

2. Eggenberger, P.. Evolving Morphologies of Simulated 3d Organisms Based on Differential Gene Expression. (Proceedings of the Fourth European Conference on Articial Life, Springer-Verlag, 1997)

3. Husbands, P., Smith, T., Jakobi, N., and O'Shea, M.. Better Living Through Chemistry: Evolving GasNets for Robot Control. Connection Science, (10)34:185-210, 1998. 